\title{
ALLOCATING FIXED RESOURCES FOR DMUS WITH INTERVAL DATA
}

\author{
Jiasen Sun ${ }^{1}$, Meng Chen ${ }^{1}$, Yelin Fu $^{2,3, *}$ and HaO LuO ${ }^{4}$
}

\begin{abstract}
Conventional DEA models tend to allocate the fixed resources to multiple decision-making units (DMUs) and treat the allocated resource as an extra input for every single DMU. However, the existing DEA resource allocation (DEA-RA) methods are applicable exclusively to the DMUs with exact values of inputs and outputs. A lack of precision for the input or output data of DMUs, such as the interval data, would cause a failure of the existing methods to allocate resources to DMUs. In order to resolve this problem, three DEA-RA models are proposed in this paper for different scenarios of decision-making. All of the proposed DEA-RA models are based on a set of common weights. Finally, the proposed models are empirically tested and validated through three examples. As revealed by the results, our proposed models are capable of providing a more fair and practical initial allocation scheme for decision makers.
\end{abstract}

Mathematics Subject Classification. 90-08, 91B32.

Received October 23, 2016. Accepted December 17, 2020.

\section{INTRODUCTION}

The resource allocation (RA) is defined as the reasonable allocation of limited resources to different departments within an individual enterprise or organization [30]. Resources are usually limited and precious, for which the reasonable and effective allocation of resources plays a significant role in the development of enterprises or organizations [4]. In addition, RA will have impact on the performance of an individual enterprise or organization [16].

The RA issue has attracted increasing attention from many scholars [14,35]. The conventional RA methods include the average allocation method, the proportional allocation method, the mathematical programming method, and the game theory [24]. Most recently, there are an increasing number of scholars using the data envelopment analysis (DEA) method to deal with the allocation of resources [36,38]. In comparison with other methods, the DEA method is advantageous as it enables a full utilization of the input and output data and removes the need for an assumption of the functional relationship between input and output [37]. In addition,

Keywords. Resource allocation, interval data, efficiency.

1 Research Center for Smarter Supply Chain, School of Business \& Dongwu Think Tank, Soochow University, No. 50, Donghuan

Road, Suzhou, RJ, China.

2 School of Intelligent Systems Science and Engineering, Jinan University (Zhuhai Campus), Zhuhai, China.

3 Department of Industrial \& Manufacturing Systems Engineering, The University of Hong Kong, Hong Kong, China.

4 College of Economics, Shenzhen University, Shenzhen, China.

* Corresponding author: msylfu@gmail.com 
by eliminating the interference of subjective factors, the final allocation results obtained from DEA are made more objective. The research of RA based on DEA can be split into two categories, which include efficiency invariant RA and efficiency variable RA [13].

The efficiency invariant RA requires that the efficiencies of the decision-making units (DMUs) remain unchanged before and after resource allocation. Cook and Kress [11] were the first to apply DEA for the cost allocation problem. They treated cost as an independent input for DMUs and then proposed a fixed cost allocation method based on DEA according to the efficiency invariance and Pareto-minimality of each DMU. However, the absence of other constraints, such as cone-ratio constraints, could prevent the model from obtaining the final optimal results. In addition, different constraints and conditions tend to produce different results, which is adverse to decision-making. In light of these problems, some scholars have examined the issue in more depth. For example, Jahanshahloo et al. [19] demonstrated that the method proposed by Cook and Kress [11] was contradictory to Pareto's minimum and then suggested a proportional method to approach cost allocation. Nevertheless, the allocation principle of the proportional method is primarily dependent on the input levels, which means, the allocation result is solely related to the input variables, with the output variables completely ignored. Apparently, this is contradictory to common sense [22]. A similar issue was encountered when using the allocation method proposed by Jahanshahloo et al. [20]. Subsequently, Cook and Zhu [12] extended the method of Cook and Kress [11] by proposing the DEA resource allocation method that took multiple inputs and multiple outputs into account. Though Cook and Zhu [12] adhered to the principle of efficiency invariance and Pareto-minimality, it was not universal. In some exceptional cases, this method was not applicable, which limited its practical applications. After this drawback was identified, scholars began to consider the efficiency and the output scale when DEA was applied to perform resource allocation. Amirteimoori and Kordrostami [3] proposed a DEA model that gave consideration to the invariance of overall average efficiency and input-output scales. By improving Cook and Zhu [12]'s method, Lin [27] proposed a new fixed cost (or resource allocation) method, which could set the output target for DMUs fairly. It was also demonstrated by the author that the proposed method could have a feasible solution when some extraordinary constraints were imposed. According to super CCR efficiency invariance and practical operability, Lin and Chen [28] adopted the DEA method to propose three models for the problems of fixed input allocation among all DMUs and common output goal sharing to be addressed.

The efficiency variable RA requires an improvement to the efficiencies of DMUs after resource allocation. Lozano and Villa [31] assumed that there was a central decision maker in the process of resource allocation and the decision maker was authorized to allocate resources or costs to all DMUs. The allocation principle is based on the minimum sum of weighted inputs or maximum sum of weighted outputs of all DMUs, for the maximum level of overall efficiency for all DMUs. Based on this principle, two centralized resource allocation models were proposed. One model seeked optimal allocation of total resources by radial technology, while the other model applied the non-radial technique to determine the optimal allocation for every type of resources depending on the preference of individual DMU. Then, the two models were further modified by Lozano et al. [32] and the modified models were applied to allocate limited container resources among major cities in Singapore for improving glass recovery. However, Asmild et al. [6] indicated that the models of Lozano and Villa [31] and Lozano et al. [32] were incapable to adjust the allocation of resources for these efficient DMUs. Subsequently, Asmild et al. [6] proposed the centralized resource allocation model based on inefficient DMUs. According to the model, both non-transferable variables and non-discretionary variables need to be taken into account. In the view of Amirteimoori and Tabar [5], if a new input resource was added into the production process, it is possible to affect the output of the DMUs. Based on this idea, Amirteimoori and Tabar [5] proposed the resource allocation and output target setting model. Based on the centralized view, Nasrabadi et al. [34] suggested that each DMU could be allowed to adjust its resources within the current production possibilities, and proposed a resource allocation DEA model for an optimal efficiency of the system. Under the common-weights scheme, Lotfi et al. [30] proposed a DEA allocation model for fixed resources, which could set targets for DMU's outputs as well. Then, the authors demonstrated the applicability of the proposed model by providing two numerical examples. Based on the studies of Lozano and Villa [31] and Asmild et al. [6], Fang [15] proposed a generalized 
centralized DEA model to approach resource allocation. Then, the proposed model was further extended by giving consideration to the non-adjustable input and non-transferable output.

These studies were invariably related to fixed resource allocation and output target identification in the centralized decision-making mode. Amirteimoori and Emrouznejad [1,2] raised a different point of view as they took into consideration the shortage of input resources in practical applications and the potential needs of decision makers to reduce the total amount of input resources at the time of resources allocation. Therefore, they proposed a DEA allocation method for the purpose of reducing inputs. Not only could the proposed model reduce the input resources as much as possible, it can also maintain the output levels and the efficiency of each DMU.

Most of these research works use a single objective function in the models, to maximize the weighted outputs of the DMU or to minimize the weighted inputs. There are other scholars who have combined the DEA method with multi-objective programming to construct the multi-objective DEA resource allocation models. Athanassopoulos [7] investigated the problems of efficiency, effectiveness and equity in resource allocation encountered by public service institutions through a diversified and multi-level framework, to achieve two major goals. One is to maximize the marginal transformation rate of input and output, and the other is to maximize the total utility of DMUs. Taking into account the ways to improve the total output from the perspective of centralization, Korhonen and Syrjanen [23] proposed a DEA model to approach resources allocation. They first described the production possibility set of DMUs, and defined the transformation possibility set. Then, the multi-objective programming and DEA were combined to propose a resource allocation scheme under different scenarios. Lozano et al. [33] adopted the DEA method for allocation of emission permits and proposed a centralized DEA emission permits allocation method, which involved three stages and goals: maximizing desirable outputs, minimizing undesirable emissions and minimizing resource consumption. Regulatory agencies or policy makers can determine the relative priority of these three goals based on their practicalities. Bi et al. [9] opened the DMU's "black box" and extended the parallel DEA method of Kao [21] to address the resource allocation and goal setting problems in parallel systems. The extended models could assist managers in allocating resources to sub-units based on their production information, thereby improving efficiency of the worst sub-unit and maintaining the efficiency of the entire production system. By treating the undesirable output as a free disposal variable, Li et al. [25] proposed two centralized resource allocation models from constant returns to scale and variable returns to scale, respectively. Hatami-Marbini et al. [17] suggested a DEA model for input or output reduction across the reference set from the perspective of centralization. In the proposed DEA model, the reduction amount of input and output for each DMU could be determined to enhance the efficiencies of all the DMUs. In consideration of the game relationship between DMUs, Li et al. [26] proposed a DEA fixed cost allocation model based on cooperative game, and took the nucleolus as the solution to the game model.

The research on DEA resource allocation is common in assuming that the data of inputs and outputs are accurate. In practice, however, this assumption is not necessarily true. In many practical circumstances, outputs and inputs of DMUs are not as precise as assumed, which may lead to a limited range in an interval form [44]. How to cope with inaccurate data has attracted increasing attention from many scholars, but a majority of the studies tend to focus on the evaluation of DMUs for their efficiencies. For example, in the case where the DMUs contained imprecise data, Zhu [46] applied the scale transformation and variable alternation methods to calculate the efficiencies of DMUs. Besides, the author proposed a simplified method to alleviate the computational workload required by the scale transformation method. To rank suppliers with imprecise data, Toloo [39] proposed an integrated mixed integer programming-DEA model based on the approach devised by Toloo and Nalchigar [41]. In addition, the author suggested a new method to rank all DMUs. In the case of dual factors and imprecise data, Toloo et al. [42] put forward two interval DEA models to obtain the interval efficiency of each DMU, and then applied the fuzzy decision-making method to obtain the final evaluation result of each DMU.

The above-mentioned literature review demonstrates that plenty of research has focused on resource allocation. However, the studies focusing on resource allocation in the case where the DMUs contain imprecise data (e.g. interval data) remain insufficient. To fill this gap, the DEA-RA approaches fit for three strategies respectively are proposed in the present study. To be specific, the first approach is based on a conservative 
strategy. The RA scheme first ensures that low efficiency of each DMU becomes efficient, and then maximizes the overall upper efficiency of all DMUs. The second RA model is based on a radical strategy. The allocation model first ensures the upper efficiency of each DMU is equal to one, and then considers the maximum level of overall lower efficiency for all DMUs. In the third model, the decision maker does not consider how to first maximize the lower or upper efficiency of each DMU. Instead, she/he may consider that it is essential to balance the gap between the lower and upper efficiencies of each DMU. This allocation strategy is defined as neutral in this study. Finally, the three cases are used to validate the effectiveness of the proposed approaches.

The rest of the paper is organized as follows. In Section 2, the traditional DEA-RA models are introduced. In Section 3, three new DEA-RA models are presented for DMUs with interval data, followed by three numerical demonstrations made in Section 4. The conclusions are indicated in Section 5.

\section{THE DEA-RA MODELS}

\subsection{Beasley [8]'s model}

It is assumed that there are $n$ independent homogeneous DMUs where each $\operatorname{DMU}_{j}(j=1, \cdots, n)$ produces $s$ different outputs $y_{r j}(r=1, \cdots, s)$ consuming $m$ inputs $x_{i j}(i=1,2, \cdots, m)$. Without any loss of generality, an assumption is made in this study that the fixed resource $R$ needs to be allocated to $n$ DMUs and the resource allocated to $\mathrm{DMU}_{j}$ is denoted as $R_{j}$.

Beasley [8] proposed a DEA-RA approach based on model (2.1), where the allocation scheme is obtained based on maximizing the average efficiency across all DMUs, with a common set of weights used.

$$
\begin{aligned}
& E_{\text {all }}^{*}=\max \frac{1}{n} \sum_{j=1}^{n} E_{j} \\
& \text { s.t. } E_{j}=\frac{\sum_{r=1}^{s} u_{r} y_{r j}}{\sum_{i=1}^{m} v_{i} x_{i j}+R_{j}} \leq 1, \quad j=1,2, \cdots, n \\
& \sum_{j=1}^{n} R_{j}=R \\
& u_{r}, v_{i}, R_{j} \geq 0, \quad \forall r, i, j .
\end{aligned}
$$

Model (2.1) is a non-linear program. In the first constraint, the weight associated with $R_{j}$ is set to one for convenience. The final allocation scheme based on model (2.1) maximizes the average efficiency of all DMUs using a common set of weights. However, model (2.1) shows three major disadvantages. Firstly, model (2.1) is a non-linear programming, which is difficult to solve. Secondly, model (2.1) is premised on the assumption that the data of all DMUs are precise. However, this assumption is not necessarily correct in practice. In many circumstances, outputs and inputs of DMUs are not as precise as expected, which may lead to a specific range in an interval form. In these cases, traditional models are not applicable to resource allocation for these DMUs. Thirdly, to solve the model (2.1), Beasley [8] proposed a solving procedure. Unfortunately, Amirteimoori and Kordrostami [3] demonstrated that the solving procedure proposed by Beasley [8] is infeasible in many cases.

\subsection{The proposed DEA-RA model for DMUs with interval data}

The focus of this paper is to design reasonable resource allocation models that take into consideration the interval data of DMUs. In the designed models, fixed resources are allocated to all DMUs on the same platform, which means, a common set of weights to all DMUs is used to deal with resource allocation. It is assumed that there are $n$ DMUs, and each DMU has $s$ different outputs and $m$ different inputs. Input $i$ and output $r$ for $\mathrm{DMU}_{j}$ are denoted as $x_{i j}$ and $y_{r j}$, respectively. The input and output data are possibly imprecise due to uncertainty, as a result of which only their bounded intervals $\left[x_{i j}^{l}, x_{i j}^{u}\right]$ and $\left[y_{r j}^{l}, y_{r j}^{u}\right]$, with $x_{i j}^{l}>0$ and $y_{r j}^{l}>0$, are provided. The fixed resource $R$ needs to be allocated to these DMUs and the allocated resource to $\mathrm{DMU}_{j}$ is 
indicated as $R_{j}$. According to the idea of Beasley [8], the resource allocation model is proposed as the following model (2.2).

$$
\begin{aligned}
& \max \sum_{j=1}^{n} \frac{\sum_{r=1}^{s} u_{r}\left[y_{r j}^{l}, y_{r j}^{u}\right]}{\sum_{i=1}^{m} v_{i}\left[x_{i j}^{l}, x_{i j}^{u}\right]+R_{j}} \\
& \text { s.t. } \frac{\sum_{r=1}^{s} u_{r}\left[y_{r j}^{l}, y_{r j}^{u}\right]}{\sum_{i=1}^{m} v_{i}\left[x_{i j}^{l}, x_{i j}^{u}\right]+R_{j}} \leq 1, \quad j=1,2, \cdots, n, \\
& \sum_{j=1}^{n} R_{j}=R \\
& v_{i}, u_{r}, R_{j} \geq 0, \quad \forall i, r .
\end{aligned}
$$

The purpose of model (2.2) is to maximize the overall efficiency of all DMUs when the fixed resource is shared by each DMU. However, it is difficult for model (2.2) to be transformed into a linear model due to the presence of interval data. Model (2.2) is extended from Beasley [8]'s model, but Beasley [8]'s solving procedure is potentially impracticable [3]. Therefore, based on Wang et al. [43]'s approach, model (2.2) is extended to the interval data DEA-RA models in this study under three strategies as referred to in Section 3.

\section{DEA-RA MOdELS UNDER THREe STRATEGies}

\subsection{DEA-RA model based on conservative strategy}

The data of each DMU is interval-valued and its efficiency is an interval efficiency as well, e.g. the efficiency of $\mathrm{DMU}_{j}$ will be $\left[E_{j}^{l}, E_{j}^{u}\right]$. If the decision maker considers it as necessary to base resource allocation on the lower bound of interval efficiency, i.e. the resource allocation scheme should ensure that lower efficiencies of all DMUs are improved to a sufficient level in the first place. Further with this, the model from the conservative strategy is constructed as follows.

$$
\begin{aligned}
& \max E_{\text {all }}^{u}=\frac{\sum_{j=1}^{n} \sum_{r=1}^{s} u_{r} y_{r j}^{u}}{\sum_{j=1}^{n}\left(\sum_{i=1}^{m} v_{i} x_{i j}^{l}+R_{j}^{u}\right)} \\
& \text { s.t. } \frac{\sum_{r=1}^{s} u_{r} y_{r j}^{l}}{\sum_{i=1}^{m} v_{i} x_{i j}^{u}+R_{j}^{u}}=1, \quad j=1,2, \cdots, n, \\
& \sum_{j=1}^{n} R_{j}^{u}=R \\
& u_{r}, v_{i}, R_{j} \geq 0, \quad \forall r, i, j .
\end{aligned}
$$

In model (3.1), the first constraint is purposed to ensure that the lower efficiency of each DMU must be efficient when total resource is allocated to all DMUs. The objective function of model (3.1) is aimed at maximizing the overall upper efficiency of all DMUs under the condition that the lower efficiency of each DMU is equal to 1.

Theorem 3.1. Model (3.1) has feasible solutions at all times.

Proof. Set $v_{i}=0(i=1,2, \cdots, m), u_{r}=0(r=1,2, \cdots, s-1), u_{s}=\frac{R}{\sum_{j=1}^{n} y_{s j}^{l}}, R_{j}^{u}=\frac{R y_{s j}^{l}}{\sum_{j=1}^{n} y_{s j}^{l}}$, then we have $\frac{\sum_{r=1}^{s} u_{r} y_{r j}^{l}}{\sum_{i=1}^{m} v_{i} x_{i j}^{u}+R_{j}^{u}}=\frac{u_{s} y_{s j}^{l}}{R_{j}^{u}}=\frac{R}{\sum_{j=1}^{n} y_{s j}^{l}} * y_{s j}^{l} / \frac{R y_{s j}^{l}}{\sum_{j=1}^{n} y_{s j}^{l}}=1, j=1,2, \cdots, n$, and $\sum_{j=1}^{n} R_{j}^{u}=\sum_{j=1}^{n} \frac{R y_{s j}^{l}}{\sum_{j=1}^{n} y_{s j}^{l}}=$ $\frac{R \sum_{j=1}^{n} y_{s j}^{l}}{\sum_{j=1}^{n} y_{s j}^{l}}=R$. Therefore, model (3.1) always has feasible solutions. 
Model (3.1) is a nonlinear model which can be transformed into a linear model via Charnes-Cooper transformation [10]. Set $\sum_{j=1}^{n}\left(\sum_{i=1}^{m} v_{i} x_{i j}^{l}+R_{j}^{u}\right)=\frac{1}{C}$, we have $C u_{r}=\mu_{r}, C v_{i}=\omega_{i}, C R_{j}^{u}=F_{j}^{u}$. Model (3.1) can be transformed into model (3.2) as follows:

$$
\begin{aligned}
& \max E_{\text {all }}^{u}=\sum_{j=1}^{n} \sum_{r=1}^{s} \mu_{r} y_{r j}^{u} \\
& \text { s.t. } \sum_{j=1}^{n}\left(\sum_{i=1}^{m} \omega_{i} x_{i j}^{l}+F_{j}^{u}\right)=1 \text {, } \\
& F_{j}^{u}=\sum_{r=1}^{s} \mu_{r} y_{r j}^{l}-\sum_{i=1}^{m} \omega_{i} x_{i j}^{u}, \quad j=1,2, \cdots, n, \\
& \sum_{j=1}^{n} F_{j}^{u}=C R \\
& \mu_{r}, \omega_{i}, F_{j}^{u} \geq 0,
\end{aligned}
$$

Theorem 3.2. In model (3.1), if any two DMUs (e.g. DMU1 and DMU2) have the same inputs $\left(x_{i 1}^{u}=x_{i 2}^{u}, i=\right.$ $1,2, \cdots, m)$, the DMU with more outputs (e.g. $\left.y_{r 1}^{l}>y_{r 2}^{l}, r=1,2, \cdots, s\right)$ will be allocated more resources.

Proof. In model (3.2), if $x_{i 1}^{u}=x_{i 2}^{u}$ and $y_{r 1}^{l}>y_{r 2}^{l}$, we have $\omega_{i} x_{i 1}^{u}=\omega_{i} x_{i 2}^{u}$ and $\mu_{r} y_{r 1}^{l}>\mu_{r} y_{r 2}^{l}$. Then $F_{1}^{u}=$ $\sum_{r=1}^{s} \mu_{r} y_{r 1}^{l}-\sum_{i=1}^{m} \omega_{i} x_{i 1}^{u}>F_{2}^{u}=\sum_{r=1}^{s} \mu_{r} y_{r 2}^{l}-\sum_{i=1}^{m} \omega_{i} x_{i 2}^{u}$, therefore, $R_{1}^{u}=\frac{F_{1}^{u}}{C}>R_{2}^{u}=\frac{F_{2}^{u}}{C}$.

A set of solutions $\left(u_{r}^{*}, v_{i}^{*}, R_{j}^{* u}\right)$ is provided by solving model (3.1). Under the conservative strategy, the upper efficiency of each DMU can be calculated as ${ }^{C} E_{j}^{u}=\frac{\sum_{r=1}^{s} u_{r}^{*} y_{r j}^{u}}{\sum_{i=1}^{m} v_{i}^{*} x_{i j}^{l}+R_{j}^{* u}}$. Based on the efficiency results, Theorem 3.3 is obtained as follows.

Theorem 3.3. Under the conservative strategy, the upper efficiency of each DMU is in excess of 1 , i.e. ${ }^{C} E_{j}^{u} \geq 1$.

Proof. From model (3.1), we know that $x_{i j}^{u} \geq x_{i j}^{l}$ and $y_{r j}^{u} \geq y_{r j}^{l}$. As $\left(u_{r}^{*}, v_{i}^{*}, R_{j}^{* u}\right)$ is a set of common solutions, we have ${ }^{C} E_{j}^{u}=\frac{\sum_{r=1}^{s} u_{r}^{*} y_{r j}^{u}}{\sum_{i=1}^{m} v_{i}^{*} x_{i j}^{l}+R_{j}^{* u}} \geq{ }^{C} E_{j}^{l}=\frac{\sum_{r=1}^{s} u_{r}^{*} y_{r j}^{l}}{\sum_{i=1}^{m} v_{i}^{*} x_{i j}^{u}+R_{j}^{* u}}$. In constraints of model (3.1), the lower efficiency of each DMU is efficient, which means ${ }^{C} E_{j}^{l}=1$, thus ${ }^{C} E_{j}^{u} \geq 1$.

\subsection{DEA-RA model based on radical strategy}

Similarly, if the decision maker believes the resource allocation scheme as based on the upper bound of interval efficiency, he/she may consider that the allocation scheme should ensure that the upper interval efficiency of each DMU is equal to one in the first place. Then, the resource allocation model obtained from the radical strategy is expressed as follows.

$$
\begin{aligned}
& \max E_{\text {all }}^{l}=\frac{\sum_{j=1}^{n} \sum_{r=1}^{s} u_{r} y_{r j}^{l}}{\sum_{j=1}^{n}\left(\sum_{i=1}^{m} v_{i} x_{i j}^{u}+R_{j}^{l}\right)} \\
& \text { s.t. } \frac{\sum_{r=1}^{s} u_{r} y_{r d}^{u}}{\sum_{i=1}^{m} v_{i} x_{i d}^{l}+R_{j}^{l}}=1, \quad j=1,2, \cdots, n \text {, } \\
& \sum_{j=1}^{n} R_{j}^{l}=R \\
& u_{r}, v_{i}, R_{j}^{l} \geq 0, \quad \forall r, i, j .
\end{aligned}
$$


Model (3.3) is a nonlinear model, which is capable to be transformed into a linear model via Charnes-Cooper transformation [10]. By setting $\sum_{j=1}^{n}\left(\sum_{i=1}^{m} v_{i} x_{i j}^{u}+R_{j}^{u}\right)=\frac{1}{C}$, we have $C u_{r}=\mu_{r}, C v_{i}=\omega_{i}, C R_{j}^{l}=F_{j}^{l}$. Model (3.3) can be transformed into model (3.4) as follows:

$$
\begin{aligned}
& \max E_{\text {all }}^{u}=\sum_{j=1}^{n} \sum_{r=1}^{s} \mu_{r} y_{r j}^{l} \\
& \text { s.t. } \sum_{j=1}^{n}\left(\sum_{i=1}^{m} \omega_{i} x_{i j}^{u}+F_{j}^{l}\right)=1 \text {, } \\
& F_{j}^{l}=\sum_{r=1}^{s} \mu_{r} y_{r d}^{u}-\sum_{i=1}^{m} \omega_{i} x_{i d}^{l}, \quad j=1,2, \cdots, n, \\
& \sum_{j=1}^{n} F_{j}^{l}=C R, \\
& \mu_{r}, \omega_{i}, F_{j}^{l} \geq 0,
\end{aligned}
$$

In model (3.3), the first constraint is proposed to ensure that the upper efficiency of each DMU is efficient when the total resource is allocated to all DMUs. The objective function of model (3.3) is aimed at maximizing the overall lower efficiency of all DMUs under the condition that the upper efficiency of each DMU is equal to 1.

Theorem 3.4. In model (3.3), if any two DMUs (e.g. DMU1 and DMU2) share the same inputs $\left(x_{i 1}^{l}=x_{i 2}^{l}, i=\right.$ $1,2, \cdots, m)$, the DMU with more outputs (e.g. $\left.y_{r 1}^{u}>y_{r 2}^{u}, r=1,2, \cdots, s\right)$ will be allocated more resource.

Proof. In model (3.4), if $x_{i 1}^{l}=x_{i 2}^{l}$ and $y_{r 1}^{u}>y_{r 2}^{u}$, we have $\omega_{i} x_{i 1}^{l}=\omega_{i} x_{i 2}^{l}$ and $\mu_{r} y_{r 1}^{u}>\mu_{r} y_{r 2}^{u}$. Then $F_{1}^{l}=$ $\sum_{r=1}^{s} \mu_{r} y_{r 1}^{u}-\sum_{i=1}^{m} \omega_{i} x_{i 1}^{l}>F_{2}^{l}=\sum_{r=1}^{s} \mu_{r} y_{r 2}^{u}-\sum_{i=1}^{m} \omega_{i} x_{i 2}^{l}$, therefore, $R_{1}^{l}=\frac{F_{1}^{l}}{C}>R_{2}^{l}=\frac{F_{2}^{l}}{C}$.

Theorem 3.5. If the efficiency score obtained from model (3.3) is 1 ( $\left.E_{\mathrm{all}}^{l}=1\right)$ each DMU based on the data $\left(x_{i j}^{u}\right.$ and $\left.y_{r j}^{l}\right)$ is treated as efficient. That is to say, the lower efficiency value of each DMU is 1.

Proof. Set $E_{\text {all }}^{l}=\frac{\sum_{j=1}^{n} \sum_{r=1}^{s} u_{r} y_{r j}^{l}}{\sum_{j=1}^{n}\left(\sum_{i=1}^{m} v_{i} x_{i j}^{u}+R_{j}^{l}\right)} R E_{j}^{l}=\frac{\sum_{r=1}^{s} u_{r} y_{r j}^{l}}{\sum_{i=1}^{m} v_{i} x_{i j}^{u}+R_{j}^{l}}, j=1, \cdots, n$ and $S=\sum_{j=1}^{n}\left(\sum_{i=1}^{m} v_{i} x_{i j}^{u}+R_{j}^{l}\right)$. We have $\frac{\sum_{j=1}^{n} \sum_{r=1}^{s} u_{r} y_{r j}^{l}}{\sum_{j=1}^{n}\left(\sum_{i=1}^{m} v_{i} x_{i j}^{u}+R_{j}^{l}\right)}=\frac{\sum_{r=1}^{s} u_{r} y_{r j}^{l}}{S} \times \frac{\sum_{i=1}^{m} v_{i} x_{i 1}^{u}+R_{1}^{l}}{\sum_{i=1}^{m} v_{i} x_{i 1}^{u}+R_{1}^{l}}+\cdots+\frac{\sum_{r=1}^{s} u_{r} y_{r n}^{l}}{S} \times \frac{\sum_{i=1}^{m} v_{i} x_{i n}^{u}+R_{n}^{l}}{\sum_{i=1}^{m} v_{i} x_{i n}^{u}+R_{n}^{l}}=\frac{\sum_{r=1}^{s} u_{r} y_{r j}^{l}}{\sum_{i=1}^{m} v_{i} x_{i 1}^{u}+R_{1}^{l}} \times$ $\frac{\sum_{i=1}^{m} v_{i} x_{i 1}^{u}+R_{1}^{l}}{S}+\cdots+\frac{\sum_{r=1}^{s} u_{r} y_{r n}^{l}}{\sum_{i=1}^{m} v_{i} x_{i n}^{u}+R_{n}^{l}} \times \frac{\sum_{i=1}^{m} v_{i} x_{i n}^{u}+R_{n}^{l}}{S}={ }^{R} E_{1}^{l} \times \frac{\sum_{i=1}^{m} v_{i} x_{i 1}^{u}+R_{1}^{l}}{S}+\cdots+{ }^{R} E_{n}^{l} \times \frac{\sum_{i=1}^{m} v_{i} x_{i n}^{u}+R_{n}^{l}}{S} E_{\text {all }}^{l}$ is the linear combination of ${ }^{R} E_{j}^{l}(j=1, \cdots, n)$, where $\frac{\sum_{i=1}^{m} v_{i} x_{i 1}^{u}+R_{1}^{l}}{S}+\cdots+\frac{\sum_{i=1}^{m} v_{i} x_{i n}^{u}+R_{n}^{l}}{S}=\frac{\sum_{j=1}^{n}\left(\sum_{i=1}^{m} v_{i} x_{i j}^{u}+R_{j}^{l}\right)}{S}=1$, $0<\frac{\sum_{i=1}^{m} v_{i} x_{i j}^{u}+R_{j}^{l}}{S} \leq 1(j=1, \cdots, n)$, and $0<{ }^{R} E_{j}^{l} \leq 1(j=1, \cdots, n)$. If one or more ${ }^{R} E_{j}^{l}$ is not equal to 1 , then $E_{\text {all }}^{l}<1$. Thus, if $E_{\text {all }}^{l}=1$, it must be met that each ${ }^{R} E_{j}^{l}$ is equal to 1 , namely ${ }^{R} E_{j}^{l}=1(j=1, \cdots, n)$.

Theorem 3.6. Under the radical strategy, the lower efficiency of each DMU is less than 1 , i.e. ${ }^{R} E_{j}^{l} \leq 1$.

Proof. From model (3.3), it can be known that $x_{i j}^{u} \geq x_{i j}^{l}$ and $y_{r j}^{u} \geq y_{r j}^{l}$. As $\left(u_{r}^{*}, v_{i}^{*}, R_{j}^{* l}\right)$ is a set of common solutions, we have ${ }^{R} E_{j}^{u}=\frac{\sum_{r=1}^{s} u_{r}^{*} y_{r j}^{u}}{\sum_{i=1}^{m} v_{i}^{*} x_{i j}^{l}+R_{j}^{* l}} \geq{ }^{R} E_{j}^{l}=\frac{\sum_{r=1}^{s} u_{r}^{*} y_{r j}^{l}}{\sum_{i=1}^{m} v_{i}^{*} x_{i j}^{u}+R_{j}^{* l}}$. In constraints of model (3.3), upper efficiency of each DMU is efficient, which means ${ }^{R} E_{j}^{u}=1$, for which ${ }^{R} E_{j}^{l} \leq 1$.

Theorem 3.7. If and only if $x_{i j}^{u}=x_{i j}^{l}$ and $y_{r j}^{u}=y_{r j}^{l}$, efficiency of model (3.3) is 1 (i.e. $E_{\text {all }}^{l}=1$ ).

Proof. From Theorem 3.5, it can be known that if $E_{\text {all }}^{l}=1$, it must be met that ${ }^{R} E_{j}^{l}=1, j=1, \cdots, n$. From Theorem 3.6, it can be obtained that ${ }^{R} E_{j}^{l} \leq 1$. Therefore, if efficiency of model (3.3) is 1 ( $E_{\text {all }}^{l}=1$ ), it must meet $x_{i j}^{u}=x_{i j}^{l}$ and $y_{r j}^{u}=y_{r j}^{l}$. 
As revealed by the comparison between models (3.1) and (3.3), the constraints of the two models are different. In model (3.3), the DMUs with the data $\left(x_{i j}^{l} y_{r j}^{u}\right.$ and $\left.R_{j}^{l}\right)$ form best-practice frontier, and the efficiency ratings of DMUs with the data $\left(x_{i j}^{u} y_{r j}^{l}\right.$ and $R_{j}^{l}$ ) could be obtained based on the best-practice frontier. In model (3.1), the DMUs with the data $\left(x_{i j}^{u} y_{r j}^{l}\right.$ and $\left.R_{j}^{u}\right)$ form a frontier, which is also called worst-practice frontier $[29,45]$. If the distance of a DMU with the data $\left(x_{i j}^{l} y_{r j}^{u}\right.$ and $\left.R_{j}^{l}\right)$ from the worst-practice frontier is increased, its efficiency is higher.

\subsection{DEA-RA model based on neutral strategy}

In consideration of the conservative and radical strategies of the DMUs, models (3.1) and (3.3) are proposed. In some cases, however, the decision maker may not pay close attention to maximizing the lower or upper efficiencies of DMUs, which is defined as a neutral strategy in this study. Considering the neutral point of view, the following neutral resource allocation model is proposed. In this model, both lower and upper efficiencies of each DMU shall be considered by the common weights and allocation scheme as constraint group (3.5).

$$
\begin{aligned}
& \text { s.t. } E_{j}^{*} \leq \frac{\sum_{r=1}^{s} u_{r} y_{r j}^{u}}{\sum_{i=1}^{m} v_{i} x_{i j}^{l}+R_{j}} \leq 1, \quad j=1,2, \cdots, n, \\
& \frac{\sum_{r=1}^{s} u_{r} y_{r j}^{l}}{\sum_{i=1}^{m} v_{i} x_{i j}^{u}+R_{j}} \leq E_{j}^{*}, \quad j=1,2, \cdots, n, \\
& \sum_{j=1}^{n} R_{j}=R \\
& u_{r}, v_{i}, R_{j} \geq 0, \quad \forall r, i, j .
\end{aligned}
$$

In model (3.3), the upper efficiency ${ }^{R} E_{j}^{* u}$ and lower efficiency ${ }^{R} E_{j}^{* l}$ of each DMU could be obtained after resource allocation. In the constraint group (3.5), it is set that $E_{j}^{*}=\frac{{ }^{R} E_{j}^{l}+{ }^{R} E_{j}^{u}}{2}$. Based on constraints group (3.5), the neutral RA model is proposed as model (3.6).

$$
\begin{aligned}
& \min \sum_{j=1}^{n} \alpha_{j}+\sum_{j=1}^{n} \beta_{j} \\
& \text { s.t. } E_{j}^{*}\left(\sum_{i=1}^{m} \omega_{i} x_{i j}^{l}+R_{j}\right)-\sum_{r=1}^{s} \mu_{r} y_{r j}^{u}+\alpha_{j}=0, \quad j=1,2, \cdots, n \text {, } \\
& E_{j}^{*}\left(\sum_{i=1}^{m} \omega_{i} x_{i j}^{u}+R_{j}\right)-\sum_{r=1}^{s} \mu_{r} y_{r j}^{l}-\beta_{j}=0, \quad j=1,2, \cdots, n, \\
& \sum_{i=1}^{m} \omega_{i} x_{i j}^{l}+R_{j}-\sum_{r=1}^{s} \mu_{r} y_{r j}^{u} \geq 0, \quad j=1,2, \cdots, n, \\
& \sum_{j=1}^{n} R_{j}=R \\
& \mu_{r}, \omega_{i}, R_{j}, \alpha_{j}, \beta_{j} \geq 0, \quad \forall r, i, j
\end{aligned}
$$

$\alpha_{j}$ and $\beta_{j}$ represent the deviations of $\mathrm{DMU}_{j}$. The objective function of model (3.6) is to minimize the sum deviations, which means, model (3.6) attempts to make the lower efficiency of each DMU approach its upper efficiency.

Theorem 3.8. If $\alpha_{j}^{*}=\beta_{j}^{*}=0$, it must be met that $x_{i j}^{u}=x_{i j}^{l}$ and $y_{r j}^{u}=y_{r j}^{l}$. 
Proof. Set $\left(\mu_{r}^{*}, \omega_{i}^{*}, R_{j}^{*}\right)$ is the solution of model (12). If $\alpha_{j}^{*}=\beta_{j}^{*}=0$, we have $\frac{\sum_{r=1}^{s} \mu_{r}^{*} y_{r j}^{u}}{\sum_{i=1}^{m} \omega_{i}^{*} x_{i j}^{l}+R_{j}^{*}}=\frac{\sum_{r=1}^{s} \mu_{r}^{*} y_{r j}^{l}}{\sum_{i=1}^{m} \omega_{i}^{*} x_{i j}^{u}+R_{j}^{*}}=$ $E_{j}^{*}(*)$. As $x_{i j}^{u} \geq x_{i j}^{l}, y_{r j}^{u} \geq y_{r j}^{l}$, if the formula $(*)$ is true, then it must be met that $x_{i j}^{u}=x_{i j}^{l}$ and $y_{r j}^{u}=y_{r j}^{l}$.

\section{ILlustrations}

In this section, three numerical examples will be provided to validate the proposed methods. The data of the three examples are presented in Tables 1, 4 and 7, respectively.

\subsection{A simple numerical example}

In Table 1, there are 10 DMUs and each DMU has 2 inputs $\left(X_{1}\right.$ and $\left.X_{2}\right)$ and 2 outputs $\left(Y_{1}\right.$ and $\left.Y_{2}\right)$. The data of inputs and outputs are all presented in the form of interval. It is assumed that the total resources are allocated for 10 DMUs $R=10$.

By comparing the results obtained from different models, we have several findings. Firstly, from Table 1, it is noted that there are two characteristics exhibited by the data of all DMUs. Firstly, input data gradually increase from DMU1 to DMU10. Secondly, output data gradually decrease from DMU1 to DMU10. If a DMU produces more outputs with less resources (inputs), it will achieve a superior performance. These findings indicate that the performance of these DMUs gradually deteriorate. Secondly, from Table 2, it can be found out that the resources allocated to all DMUs gradually diminish from DMU1 to DMU10, which suggests that, if an individual DMU produces more outputs, it is supposed to be capable of obtaining more resources. For instance DMU5

TABLE 1. A simple numerical example.

\begin{tabular}{lllll}
\hline \hline DMU & $X_{1}$ & $X_{2}$ & $Y_{1}$ & $Y_{2}$ \\
\hline 1 & {$[1,2]$} & {$[2,3]$} & {$[23,24]$} & {$[22,24]$} \\
2 & {$[2,3]$} & {$[4,4]$} & {$[21,22]$} & {$[22,23]$} \\
3 & {$[3,4]$} & {$[5,6]$} & {$[20,21]$} & {$[20,21]$} \\
4 & {$[3,4]$} & {$[5,7]$} & {$[16,18]$} & {$[19,19]$} \\
5 & {$[3,5]$} & {$[5,7]$} & {$[14,17]$} & {$[13,15]$} \\
6 & {$[4,5]$} & {$[6,7]$} & {$[12,15]$} & {$[10,14]$} \\
7 & {$[4,5]$} & {$[7,8]$} & {$[10,15]$} & {$[9,14]$} \\
8 & {$[4,6]$} & {$[8,8]$} & {$[9,14]$} & {$[8,13]$} \\
9 & {$[5,6]$} & {$[8,9]$} & {$[9,14]$} & {$[8,13]$} \\
10 & {$[5,7]$} & {$[8,9]$} & {$[8,12]$} & {$[7,13]$} \\
\hline
\end{tabular}

TABLE 2. The cost allocation results of all DMUs from three models.

\begin{tabular}{llll}
\hline \hline DMU & Model (3.1) & Model (3.3) & Model (3.6) \\
\hline 1 & 2.3463 & 1.3953 & 1.2942 \\
2 & 1.9903 & 1.2791 & 1.1886 \\
3 & 1.7476 & 1.2209 & 1.1359 \\
4 & 1.2945 & 1.0465 & 1.0063 \\
5 & 0.9385 & 0.9884 & 0.9845 \\
6 & 0.7120 & 0.8721 & 0.8800 \\
7 & 0.4854 & 0.8721 & 0.9504 \\
8 & 0.2427 & 0.8140 & 0.8999 \\
9 & 0.2427 & 0.8140 & 0.8999 \\
10 & 0.0000 & 0.6977 & 0.7603 \\
\hline
\end{tabular}


TABLE 3. The interval efficiency results of all DMUs from three models.

\begin{tabular}{|c|c|c|c|c|c|c|c|c|}
\hline \multirow[t]{2}{*}{ DMU } & \multicolumn{2}{|c|}{ Wang et al. [43] } & \multicolumn{2}{|c|}{ Model (3.1) } & \multicolumn{2}{|c|}{ Model (3.3) } & \multicolumn{2}{|c|}{ Model (3.6) } \\
\hline & $\begin{array}{l}\text { Lower } \\
\text { efficiency }\end{array}$ & $\begin{array}{l}\text { Upper } \\
\text { efficiency }\end{array}$ & $\begin{array}{l}\text { Lower } \\
\text { efficiency }\end{array}$ & $\begin{array}{l}\text { Upper } \\
\text { efficiency }\end{array}$ & $\begin{array}{l}\text { Lower } \\
\text { efficiency }\end{array}$ & $\begin{array}{l}\text { Upper } \\
\text { efficiency }\end{array}$ & $\begin{array}{l}\text { Lower } \\
\text { efficiency }\end{array}$ & $\begin{array}{l}\text { Upper } \\
\text { efficiency }\end{array}$ \\
\hline 1 & 0.6389 & 1.0000 & 1.0000 & 1.0980 & 0.9583 & 1.0000 & 0.9384 & 0.9792 \\
\hline 2 & 0.4583 & 0.4792 & 1.0000 & 1.1079 & 0.9545 & 1.0000 & 0.9329 & 0.9773 \\
\hline 3 & 0.2778 & 0.3500 & 1.0000 & 1.1136 & 0.9524 & 1.0000 & 0.9297 & 0.9762 \\
\hline 4 & 0.2262 & 0.3167 & 1.0000 & 1.2115 & 0.8889 & 1.0000 & 0.8395 & 0.9444 \\
\hline 5 & 0.1667 & 0.2833 & 1.0000 & 1.4512 & 0.8235 & 1.0000 & 0.7509 & 0.9118 \\
\hline 6 & 0.1429 & 0.2083 & 1.0000 & 1.3816 & 0.8000 & 1.0000 & 0.7200 & 0.9000 \\
\hline 7 & 0.1042 & 0.1786 & 1.0000 & 1.6935 & 0.6667 & 1.0000 & 0.5556 & 0.8333 \\
\hline 8 & 0.0937 & 0.1458 & 1.0000 & 2.0851 & 0.6429 & 1.0000 & 0.5281 & 0.8214 \\
\hline 9 & 0.0833 & 0.1458 & 1.0000 & 1.7818 & 0.6429 & 1.0000 & 0.5281 & 0.8214 \\
\hline 10 & 0.0741 & 0.1354 & 1.0000 & 2.1000 & 0.6667 & 1.0000 & 0.5556 & 0.8333 \\
\hline
\end{tabular}

TABLE 4. The data of input and output of all schools.

\begin{tabular}{llllll}
\hline \hline School & $\begin{array}{l}\text { Number } \\
\text { of staff }\end{array}$ & $\begin{array}{l}\text { School building area } \\
\text { (Square meters) }\end{array}$ & $\begin{array}{l}\text { Copies of } \\
\text { books }\end{array}$ & $\begin{array}{l}\text { Fixed asset } \\
\text { (Million } \\
\text { RMB) }\end{array}$ & $\begin{array}{l}\text { Number of } \\
\text { students }\end{array}$ \\
\hline 1 & {$[47,53]$} & 3964 & 8947 & 3.54 & {$[313,360]$} \\
2 & {$[39,40]$} & 965 & 4247 & 2.04 & {$[102,110]$} \\
3 & {$[65,70]$} & 2222 & 8543 & 2.23 & {$[263,300]$} \\
4 & {$[43,54]$} & 2316 & 7560 & 2.42 & {$[261,274]$} \\
5 & {$[47,49]$} & 3362 & 11035 & 1.23 & {$[292,312]$} \\
6 & {$[49,59]$} & 3273 & 6120 & 5.61 & {$[261,289]$} \\
7 & {$[30,36]$} & 1534 & 7439 & 2.55 & {$[256,270]$} \\
8 & {$[45,57]$} & 1130 & 4043 & 2.25 & {$[73,81]$} \\
9 & {$[38,45]$} & 2278 & 7306 & 1.51 & {$[293,311]$} \\
10 & {$[104,124]$} & 7321 & 25218 & 16.91 & {$[1129,1195]$} \\
11 & {$[92,110]$} & 6218 & 11552 & 10.86 & {$[410,455]$} \\
12 & {$[38,40]$} & 1878 & 4155 & 3.89 & {$[191,202]$} \\
13 & {$[42,46]$} & 2649 & 6986 & 1.41 & {$[242,263]$} \\
14 & {$[39,50]$} & 2402 & 8623 & 2.18 & {$[264,341]$} \\
15 & {$[55,57]$} & 2359 & 7200 & 5.06 & {$[221,264]$} \\
16 & {$[30,39]$} & 1328 & 6260 & 1.87 & {$[179,227]$} \\
17 & {$[132,137]$} & 11922 & 53840 & 8.28 & {$[2672,3122]$} \\
18 & {$[59,62]$} & 3552 & 11674 & 6.76 & {$[417,505]$} \\
19 & {$[17,19]$} & 1666 & 3926 & 2.98 & {$[125,147]$} \\
20 & {$[173,180]$} & 23200 & 40000 & 23.09 & {$[3066,3122]$} \\
21 & {$[73,74]$} & 3271 & 21484 & 2.34 & {$[360,386]$} \\
22 & {$[59,72]$} & 4301 & 10300 & 2.26 & {$[290,363]$} \\
23 & {$[99,112]$} & 21175 & 47060 & 7.34 & {$[1995,2317]$} \\
24 & {$[35,41]$} & 1410 & 13803 & 1.65 & {$[212,230]$} \\
25 & {$[65,105]$} & 30705 & 22000 & 38.30 & {$[1252,1276]$} \\
\hline & & & & & \\
\hline
\end{tabular}

and DMU6 have the same inputs $\left(x_{i 1}^{u}=x_{i 2}^{u}\right)$ and the DMU5 with more outputs (e.g. $\left.y_{r 1}^{l}>y_{r 2}^{l}\right)$ is allocated more resources. Thirdly, Table 3 shows the efficiencies of all DMUs before resource allocation, using the interval efficiency model of Wang et al. [43]. Comparing the efficiency results obtained from our proposed models and that of Wang et al. [43], it is noted that the efficiency of each DMU improves after resource allocation by 
TABLE 5. The allocation results of all schools.

\begin{tabular}{lllll}
\hline \hline School & Original allocation & Model (3.1) & Model (3.3) & Model (3.6) \\
\hline 1 & 9.26 & 4.65 & 4.92 & 5.37 \\
2 & 3.41 & 0.96 & 1.31 & 1.59 \\
3 & 12.07 & 3.29 & 4.35 & 4.46 \\
4 & 5.70 & 3.64 & 3.88 & 3.91 \\
5 & 5.90 & 4.35 & 4.14 & 4.49 \\
6 & 8.53 & 3.52 & 3.73 & 4.23 \\
7 & 5.73 & 3.98 & 4.01 & 3.87 \\
8 & 10.07 & 0.00 & 0.67 & 1.19 \\
9 & 7.60 & 4.46 & 4.77 & 4.47 \\
10 & 15.73 & 18.39 & 17.80 & 17.14 \\
11 & 13.95 & 5.10 & 5.17 & 6.67 \\
12 & 6.43 & 2.65 & 2.74 & 2.90 \\
13 & 6.22 & 3.47 & 3.74 & 3.82 \\
14 & 7.25 & 3.79 & 5.13 & 5.36 \\
15 & 8.57 & 2.81 & 3.42 & 4.01 \\
16 & 5.68 & 2.45 & 3.39 & 3.54 \\
17 & 20.07 & 47.33 & 53.59 & 46.91 \\
18 & 8.20 & 6.40 & 7.33 & 7.71 \\
19 & 2.83 & 1.91 & 1.80 & 2.22 \\
20 & 25.18 & 53.76 & 50.38 & 43.93 \\
21 & 10.90 & 5.03 & 4.46 & 5.57 \\
22 & 10.14 & 3.75 & 4.88 & 5.63 \\
23 & 14.35 & 35.11 & 35.58 & 34.72 \\
24 & 5.37 & 3.02 & 2.67 & 3.34 \\
25 & 15.99 & 21.19 & 11.15 & 17.96 \\
\hline & & & & \\
\hline
\end{tabular}

applying our models, which indicates that our models allocate resources to each DMU from the perspective of resource utilization efficiency. Finally, as indicated by Table 2, when different preferences are given to the lower or upper bound of the interval, the final allocation results are different. If the decision maker would like to allocate resource to DMUs with interval data, the first thing to do is to determine which bound (lower or upper) is of more importance.

\subsection{The case of primary schools}

The illustrative application involves a dataset of all primary schools located in China's Jinhu County, Jiangsu, China. To be consistent with extant studies in literature, the number of staff, school building area (in Square meters), copies of books and fixed assets (in $10^{6}$ RMB Yuan) are taken as input variables. The number of students in each school is taken as the output variable. All the data is collected from the Education Bureau of Jinhu County. The school profile is shown in Table 4 . The total budget for these schools is 245 (in $10^{6}$ RMB Yuan).

Table 4 indicates the significant differences in inputs and outputs of these primary schools, and the maximum value is shown to be 31 times higher than the minimum value. The differences between variables of school building area, fixed assets and number of students among schools are found substantial. Of these indicators, the number of staff and the number of students are indicated in an interval form. As the staff and students might quit the school or move from one school to another, these data are not fixed and are collected from the beginning and end of the year, in an interval form.

The second column of Table 5 shows the allocation results obtained from the local government. The second to fifth columns of Table 6 present the efficiency results before and after government allocation. The comparison performed among the results obtained from different models leads to a number of findings. 


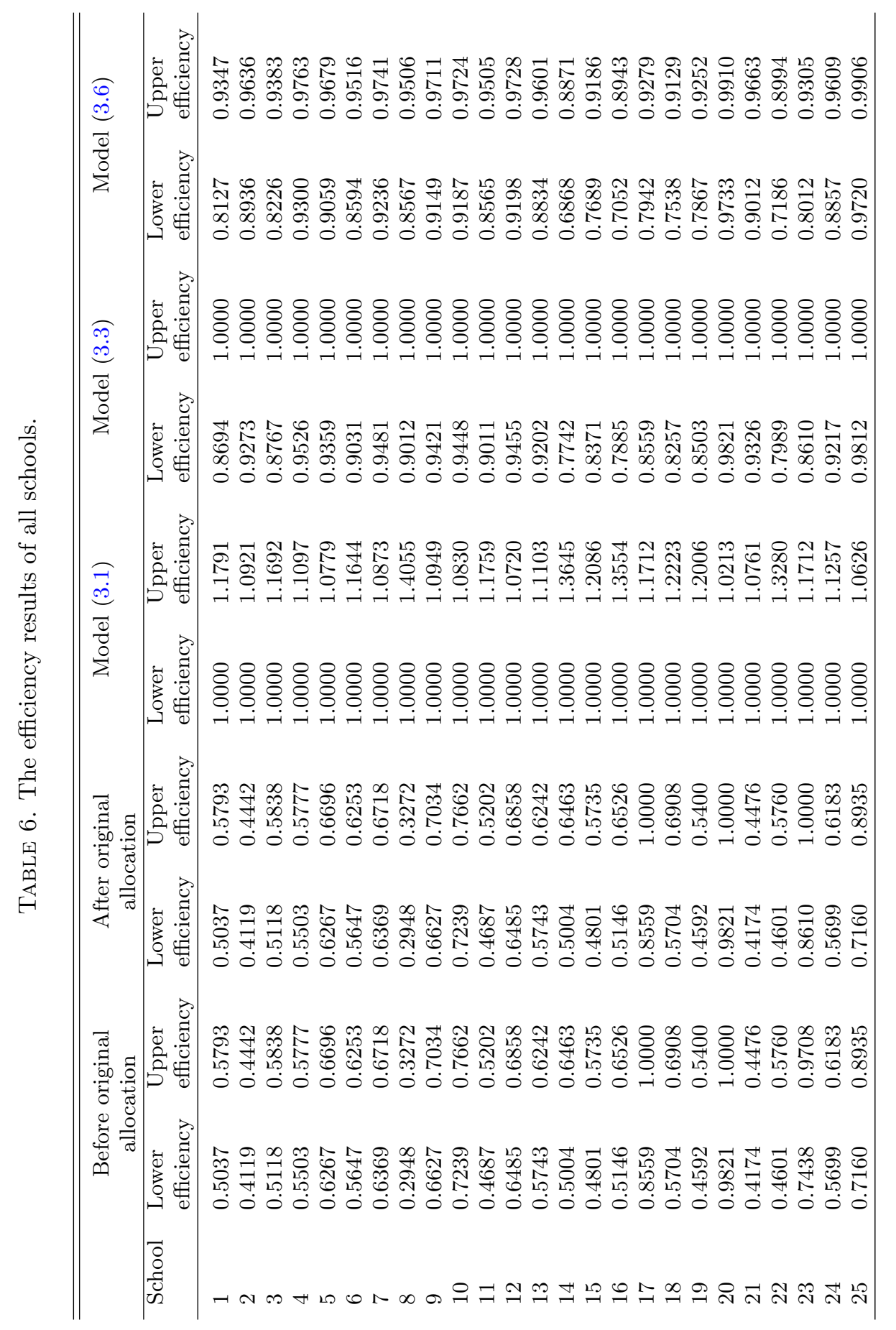


Firstly, by comparing the efficiencies of DMUs before and after the original allocation, it can be known that there is no significant improvement to efficiency for most schools, which indicates that the original allocation scheme from the perspective of the government does not take into account of the efficiency of resource utilization.

Secondly, it is worthy that the efficiency of each school after budget allocation using any one of the proposed models is significantly higher than before allocation which suggests that our proposed models will improve efficiency to the highest possible level for each school, for the full utilization of educational resources.

Thirdly, Table 5 indicates that there are numerous instances of unreasonable allocations of government funds. For example, School 2 educates a small number of students with a substantial amount of educational resources. In other words, the educational resources allocated to School 2 fail to be fully utilized. Thus, what the government needs to do is finding ways to assist School 2 with the overall planning and full utilization of the existing educational resources. Actually, however, the government allocates 1207 million RMB to School 2, which does not appear in our models as our models allocate 329 million RMB, 435 million RMB and 446 million RMB to School 2, respectively. The resource allocation scheme of our method is primarily based on the efficiency of DMU. The efficiency of school 10 before the resource allocation is [0.7239 0.7662$]$, higher compared with other similar-sized schools. Therefore, school 10 obtains more resources than other similar-sized school.

Finally, three types of allocation results are found to be different, which is because each proposed model adopts a different strategy. Therefore, the decision maker could be allowed flexibility in the selection of the appropriate model based on the practicalities

\subsection{The case of Hatami-Marbini et al. [18]}

Table 7 shows the interval data for the case of 12 DMUs, which comes from Hatami-Marbini et al. [18]. In this section, the results of Hatami-Marbini et al. [18]'s approach and our proposed approach will be compared through these 12 DMUs. As for the data, Hatami-Marbini et al. [18] assumed that the upper bound of each input (or output) is 1.105 times the lower bound, and the total resources to be allocated are interval data as well $(R=[90,110])$. However, our approach is based on the assumption that the total resources are fixed. In this section, the total resources to be allocated in our approach are set to 110 .

Table 8 shows the calculation results of the two methods, which leads to three major findings. Firstly, HatamiMarbini et al. [18] assumed that the total resources are the interval data $(R=[90,110])$, and the allocation result is in the form of interval as well. The results in the interval forms may still make the decision-makers confused over how to allocate resources. However, this problem is avoided in our approach, and a set of accurate

TABLE 7. The case of 12 DMUs from Hatami-Marbini et al. [18].

\begin{tabular}{lllllllllll}
\hline \hline \multirow{2}{*}{ DMU } & \multicolumn{3}{c}{ Input 1 } & \multicolumn{2}{c}{ Input 2 } & \multicolumn{2}{c}{ Input 3 } & \multicolumn{2}{c}{ Output 1 } & \multicolumn{2}{c}{ Output 2 } \\
\cline { 2 - 11 } & $\begin{array}{l}\text { Lower } \\
\text { bound }\end{array}$ & $\begin{array}{l}\text { Upper } \\
\text { bound }\end{array}$ & $\begin{array}{l}\text { Lower } \\
\text { bound }\end{array}$ & $\begin{array}{l}\text { Upper } \\
\text { bound }\end{array}$ & $\begin{array}{l}\text { Lower } \\
\text { bound }\end{array}$ & $\begin{array}{l}\text { Upper } \\
\text { bound }\end{array}$ & $\begin{array}{c}\text { Lower } \\
\text { bound }\end{array}$ & $\begin{array}{l}\text { Upper } \\
\text { bound }\end{array}$ & $\begin{array}{c}\text { Lower } \\
\text { bound }\end{array}$ & $\begin{array}{l}\text { Upper } \\
\text { bound }\end{array}$ \\
\hline 1 & 332.5 & 367.5 & 37.05 & 40.95 & 8.55 & 9.45 & 63.65 & 70.35 & 713.45 & 788.55 \\
2 & 283.1 & 312.9 & 24.7 & 27.3 & 7.6 & 8.4 & 69.35 & 76.65 & 580.45 & 641.55 \\
3 & 400.9 & 443.1 & 29.45 & 32.55 & 6.65 & 7.35 & 71.25 & 78.75 & 554.8 & 613.2 \\
4 & 266.95 & 295.05 & 15.2 & 16.8 & 8.55 & 9.45 & 66.5 & 73.5 & 631.75 & 698.25 \\
5 & 285.95 & 316.05 & 15.2 & 16.8 & 5.7 & 6.3 & 71.25 & 78.75 & 422.75 & 467.25 \\
6 & 342 & 378 & 27.55 & 30.45 & 16.15 & 17.85 & 78.85 & 87.15 & 1016.5 & 1123.5 \\
7 & 513 & 567 & 17.1 & 18.9 & 9.5 & 10.5 & 68.4 & 75.6 & 434.15 & 479.85 \\
8 & 262.2 & 289.8 & 31.35 & 34.65 & 4.75 & 5.25 & 74.1 & 81.9 & 560.5 & 619.5 \\
9 & 306.85 & 339.15 & 23.75 & 26.25 & 4.75 & 5.25 & 71.25 & 78.75 & 1020.3 & 1127.7 \\
10 & 421.8 & 466.2 & 60.8 & 67.2 & 5.7 & 6.3 & 70.3 & 77.7 & 1018.4 & 1125.6 \\
11 & 306.85 & 339.15 & 23.75 & 26.25 & 4.75 & 5.25 & 23.75 & 26.25 & 332.5 & 367.5 \\
12 & 421.8 & 466.2 & 60.8 & 67.2 & 5.7 & 6.3 & 98.8 & 109.2 & 1139.05 & 1258.95 \\
\hline
\end{tabular}


TABLE 8. The results of two approaches.

\begin{tabular}{|c|c|c|c|c|c|c|c|}
\hline \multirow[t]{2}{*}{ DMU } & \multicolumn{2}{|c|}{$\begin{array}{l}\text { Hatami-Marbini } \\
\text { et al. [18]'s RA } \\
\text { approach }\end{array}$} & \multicolumn{3}{|c|}{$\begin{array}{l}\text { Hatami-Marbini } \\
\text { et al. }[18] \text { 's Efficiency } \\
\text { evaluation approach }\end{array}$} & \multicolumn{2}{|c|}{$\begin{array}{l}\text { Our approach } \\
\text { Model (3.3) }\end{array}$} \\
\hline & $R_{j}^{l *}$ & $R_{j}^{u *}$ & $\begin{array}{l}\text { Lower } \\
\text { efficiency }\end{array}$ & $\begin{array}{l}\text { Upper } \\
\text { efficiency }\end{array}$ & $R_{j}^{*}$ & $\begin{array}{l}\text { Lower } \\
\text { efficiency }\end{array}$ & $\begin{array}{l}\text { Upper } \\
\text { efficiency }\end{array}$ \\
\hline 1 & 7.2855 & 7.2855 & 0.9847 & 1.0000 & 9.2587 & 0.9048 & 1.0000 \\
\hline 2 & 6.6510 & 6.6510 & 1.0000 & 1.0000 & 7.6881 & 0.9048 & 1.0000 \\
\hline 3 & 3.7643 & 9.2516 & 0.7083 & 1.0000 & 7.3923 & 0.9048 & 1.0000 \\
\hline 4 & 8.5474 & 8.5474 & 1.0000 & 1.0000 & 8.2881 & 0.9048 & 1.0000 \\
\hline 5 & 4.2810 & 4.2810 & 1.0000 & 1.0000 & 5.7829 & 0.9048 & 1.0000 \\
\hline 6 & 14.1509 & 14.1509 & 0.9959 & 1.0000 & 13.0867 & 0.9048 & 1.0000 \\
\hline 7 & 0.1484 & 4.5606 & 0.6877 & 1.0000 & 5.8966 & 0.9048 & 1.0000 \\
\hline 8 & 6.3419 & 7.4261 & 0.9416 & 1.0000 & 7.4870 & 0.9048 & 1.0000 \\
\hline 9 & 15.0964 & 15.0964 & 0.9985 & 1.0000 & 13.0658 & 0.9048 & 1.0000 \\
\hline 10 & 10.2222 & 12.1773 & 0.9070 & 1.0000 & 13.0342 & 0.9048 & 1.0000 \\
\hline 11 & 0 & 7.0612 & 0.4946 & 1.0000 & 4.2626 & 0.9048 & 1.0000 \\
\hline \multirow[t]{3}{*}{12} & 13.5110 & 13.5110 & 1.0000 & 1.0000 & 14.7569 & 0.9048 & 1.0000 \\
\hline & (Sum) & (Sum) & (Ave.) & (Ave.) & (Sum) & (Ave.) & (Ave.) \\
\hline & 90 & 110 & 0.8932 & 1.0000 & 110 & 0.9048 & 1.0000 \\
\hline
\end{tabular}

allocation results are obtained. Secondly, in Hatami-Marbini et al. [18]'s results, the lower bound efficiencies of DMUs show a significant fluctuation, and the average value is lower compared with our approach. Thirdly, in the data of case, Hatami-Marbini et al. [18] assumed that the upper bound of each input (or output) is 1.105 times the lower bound. According to the DEA axiom, the input and output of a DMU increase (or decrease) simultaneously by the same multiple, and its efficiency will remain unchanged. After resource allocation, a new input is added to each DMU. The upper efficiency and lower efficiency of each DMU by our approach are 1 and 0.9048 , respectively, which conforms to the characteristics of case data and DEA axiom.

\section{CONCLUSiOn}

DEA has played a significant role in the performance evaluation and benchmarking since it was first proposed. Recently, scholars have extended DEA to the allocation of fixed resource for a group of DMUs. The existing DEARA models are premised on the assumption that the data of all DMUs are precise. However, this assumption is not necessarily correct in practice. Under many circumstances, the outputs and inputs of DMUs are not as precise as assumed, i.e. the data are only in a range in an interval form. In these cases, the existing DEA models cannot be applicable to resource allocation. In order to resolve this problem, three DEA resource allocation models with different strategies are proposed in this study. The first model is based on a conservative strategy, while the second model is based on a radical strategy. In the resource allocation models, the conservative (radical) strategy first ensures the lower (upper) efficiencies of all DMUs to be efficient and then maximizes the overall upper (lower) efficiency of all DMUs. The third model is based on a neutral strategy, i.e. resource allocation scheme pays attention to reducing the gap between the lower and the upper efficiencies of each DMU in the first place. The proposed models are illustrated and validated by introducing three demonstrative cases. It is concluded that the proposed approaches are effective in resources allocation to DMUs with interval data, and are capable to produce fair results for all DMUs.

This study features the proposal of three DEA resource allocation models for DMUs with interval data. In the future these models could be extended by taking a robust optimization DEA approach proposed by Toloo and Mensah [40]. In addition, this study could be extended for resource allocation of DMUs with random data (or fuzzy data) in future research. 
Acknowledgements. This research is partially supported by the National Natural Science Foundation of China under the grant Nos. 71871153, 71501139 and 71671116; the National Social Science Foundation of China (No. 19BGL188); the Guangdong Basic and Applied Basic Research Foundation (No. 2019A1515111164). Jiasen Sun thanks the sponsorship of Jiangsu Overseas Visiting Scholar Program for University Prominent Young \& Middle-aged Teachers and Presidents.

\section{REFERENCES}

[1] A. Amirteimoori and A. Emrouznejad, Input/output deterioration in production processes. Expert Syst. App. 38 (2011) 58225825 .

[2] A. Amirteimoori and A. Emrouznejad, Optimal input/output reduction in production processes. Decis. Support Syst. 52 (2012) $742-747$.

[3] A. Amirteimoori and S. Kordrostami, Allocating fixed costs and target setting: a DEA-based approach. Appl. Math. Comput. 171 (2005) 136-151.

[4] A. Amirteimoori and M. Shafiei, Characterizing an equitable omission of shared resources: a DEA-based approach. Appl. Math. Comput. 177 (2006) 18-23.

[5] A. Amirteimoori and M.M. Tabar, Resource allocation and target setting in data envelopment analysis. Expert Syst. App. 37 (2010) 3036-3039.

[6] M. Asmild, J.C. Paradi and J.T. Pastor, Centralized resource allocation BCC models. Omega 37 (2009) $40-49$.

[7] A.D. Athanassopoulos, Decision support for target-based resource allocation of public services in multiunit and multilevel systems. Manage. Sci. 44 (1998) 173-187.

[8] J.E. Beasley, Allocating fixed costs and resources via data envelopment analysis. Eur. J. Oper. Res. 147 (2003) $198-216$.

[9] G. Bi, J. Ding, Y. Luo and L. Liang, Resource allocation and target setting for parallel production system based on DEA. Appl. Math. Modell. 35 (2011) 4270-4280.

[10] A. Charnes and W.W. Cooper, Programming with linear fractional functionals. Nav. Res. Logistics Q. 9 (1962) $181-186$.

[11] W.D. Cook and M. Kress, Characterizing an equitable allocation of shared costs: a DEA approach. Eur. J. Oper. Res. 119 (1999) 652-661.

[12] W.D. Cook and J. Zhu, Allocation of shared costs among decision making units: a DEA approach. Comput. Oper. Res. 32 (2005) 2171-2178.

[13] T. Ding, Y. Chen, H. Wu and Y. Wei, Centralized fixed cost and resource allocation considering technology heterogeneity: a DEA approach. Ann. Oper. Res. 268 (2018) 497-511.

[14] A. Emrouznejad and G.L. Yang, A survey and analysis of the first 40 years of scholarly literature in DEA: 1978-2016. Soc.-Econ. Planning Sci. 61 (2018) 4-8.

[15] L. Fang, A generalized DEA model for centralized resource allocation. Eur. J. Oper. Res. 228 (2013) $405-412$.

[16] B. Golany and E. Tamir, Evaluating efficiency-effectiveness-equality trade-offs: a data envelopment analysis approach. Manage. Sci. 41 (1995) 1172-1184.

[17] A. Hatami-Marbini, M. Tavana, P.J. Agrell, F.H. Lotfi and Z.G. Beigi, A common-weights DEA model for centralized resource reduction and target setting. Comput. Ind. Eng. 79 (2015) 195-203.

[18] A. Hatami-Marbini, Z.G. Beigi, H. Fukuyama and K. Gholami, Modeling centralized resources allocation and target setting in imprecise data envelopment analysis. Int. J. Inf. Technol. Decis. Making 14 (2015) 1189-1213.

[19] G.R. Jahanshahloo, F.H. Lotfi, N. Shoja and M. Sanei, An alternative approach for equitable allocation of shared costs by using DEA. Appl. Math. Comput. 153 (2004) 267-274.

[20] G.R. Jahanshahloo, F.H. Lotfi and M. Moradi, A DEA approach for fair allocation of common revenue. Appl. Math. Comput. 160 (2005) 719-724.

[21] C. Kao, Efficiency measurement for parallel production systems. Eur. J. Oper. Res. 196 (2009) 1107-1112.

[22] M. Khodabakhshi and K. Aryavash, The fair allocation of common fixed cost or revenue using DEA concept. Ann. Oper. Res. 214 (2014) 187-194.

[23] P. Korhonen and M. Syrjänen, Resource allocation based on efficiency analysis. Manage. Sci. 50 (2004) $1134-1144$.

[24] Y. Li, Research on fixed cost allocation method based on DEA Theory. Doctoral dissertation, University of Science and Technology of China, Hefei (2008).

[25] H. Li, W. Yang, Z. Zhou and C. Huang, Resource allocation models' construction for the reduction of undesirable outputs based on DEA methods. Math. Comput. Modell. 58 (2013) 913-926.

[26] Y. Li, F. Li, A. Emrouznejad, L. Liang and Q. Xie, Allocating the fixed cost: an approach based on data envelopment analysis and cooperative game. Ann. Oper. Res. 274 (2019) 373-394.

[27] R. Lin, Allocating fixed costs or resources and setting targets via data envelopment analysis. Appl. Math. Comput. 217 (2011) 6349-6358.

[28] R. Lin and Z. Chen, Fixed input allocation methods based on super CCR efficiency invariance and practical feasibility. Appl. Math. Modell. 40 (2016) 5377-5392.

[29] F.H.F. Liu and C.L. Chen, The worst-practice DEA model with slack-based measurement. Comput. Ind. Eng. 57 (2009) 496-505.

[30] F.H. Lotfi, A. Hatami-Marbini, P.J. Agrell, N. Aghayi and K. Gholami, Allocating fixed resources and setting targets using a common-weights DEA approach. Comput. Ind. Eng. 64 (2013) 631-640. 
[31] S. Lozano and G. Villa, Centralized resource allocation using data envelopment analysis. J. Prod. Anal. 22 (2004) $143-161$.

[32] S. Lozano, G. Villa and B. Adenso-Diaz, Centralised target setting for regional recycling operations using DEA. Omega 32 (2004) 101-110.

[33] S. Lozano, G. Villa and R. Brännlund, Centralised reallocation of emission permits using DEA. Eur. J. Oper. Res. 193 (2009) $752-760$.

[34] N. Nasrabadi, A. Dehnokhalaji, N.A. Kiani, P.J. Korhonen and J. Wallenius, Resource allocation for performance improvement. Ann. Oper. Res. 196 (2012) 459-468.

[35] J. Sun and G. Li, Designing a double auction mechanism for the re-allocation of emission permits. Ann. Oper. Res. 291 (2020) $847-874$.

[36] J. Sun, Y. Fu, X. Ji and R.Y. Zhong, Allocation of emission permits using DEA-game-theoretic model. Oper. Res. 17 (2017) $867-884$.

[37] J. Sun, C. Wang, X. Ji and J. Wu, Performance evaluation of heterogeneous bank supply chain systems from the perspective of measurement and decomposition. Comput. Ind. Eng. 113 (2017) 891-903.

[38] J. Sun, G. Li and Z. Wang, Optimizing China's energy consumption structure under energy and carbon constraints. Struct. Change Econ. Dyn. 47 (2018) 57-72.

[39] M. Toloo, Selecting and full ranking suppliers with imprecise data: a new DEA method. The Int. J. Adv. Manuf. Technol. 74 (2014) 1141-1148.

[40] M. Toloo and E.K. Mensah, Robust optimization with nonnegative decision variables: a DEA approach. Comput. Ind. Eng. 127 (2019) 313-325.

[41] M. Toloo and S. Nalchigar, A new DEA method for supplier selection in presence of both cardinal and ordinal data. Expert Syst. App. 38 (2011) 14726-14731.

[42] M. Toloo, E. Keshavarz and A. Hatami-Marbini, Dual-role factors for imprecise data envelopment analysis. Omega 77 (2018) $15-31$.

[43] Y.M. Wang, R. Greatbanks and J.B. Yang, Interval efficiency assessment using data envelopment analysis. Fuzzy Sets Syst. 153 (2005) 347-370.

[44] J. Wu, J. Sun, M. Song and L. Liang, A ranking method for DMUs with interval data based on DEA cross-efficiency evaluation and TOPSIS. J. Syst. Sci. Syst. Eng. 22 (2013) 191-201.

[45] F. Yang, F. Du, L. Liang and L. Ling, Full ranking procedure based on best and worst frontiers. J. Syst. Eng. Electron. 26 (2015) 514-522.

[46] J. Zhu, Imprecise data envelopment analysis (IDEA): a review and improvement with an application. Eur. J. Oper. Res. 144 (2003) 513-529. 